\title{
Game Adaptivity Impact on Affective Physical Interaction
}

\author{
Georgios N. Yannakakis \\ Center for Computer Games Research \\ IT University of Copenhagen \\ Rued Langgaards Vej 7, DK-2300 Copenhagen S \\ yannakakiseitu.dk
}

\begin{abstract}
Adaptive human computer interaction is necessary for successfully closing the affective loop within intelligent interactive systems. This paper investigates the impact of adaptivity on the physiological state and the expressed emotional preferences of users. A physical interactive game is used as a test-bed system and its real-time adaptation mechanism is evaluated using a survey experiment. Results reveal that entertainment preferences expressed are consistent with the affective model constructed and that adaptation generates dissimilar physiological responses with respect to preferences.
\end{abstract}

\section{Introduction}

There is an increasing research interest towards personalization of human machine interaction and construction of adaptive systems built on computational models of user interaction. The successful realization of the biocybernetic loop [17] as the core component of a physiological computing system defines an important pathway toward such adaptive systems. Motivated by the lack of analysis on the impact of adaptation on constructed affective models and reported emotions this paper investigates the effect of adaptivity to physiology and expressed emotional preferences in physical interactive play. The Playware [12] physical activity platform is used as a test-bed for the experiments presented here.

An artificial neural network (ANN) user model has been constructed [24] that efficiently maps between controllable Playware game characteristics (game features), individual playing behavior statistical data (player features) and expressed entertainment ("fun") preferences - entertainment and fun will be used interchangeably in this paper. Following the same approach of preference learning, an accurate ANN model of entertainment preferences has been built on physiological signal data [26]. The former and the latter model are named, respectively, interaction and physiological model of reported entertainment; both models output numerical values for entertainment that successfully match the entertainment preferences of users. Data used for training the ANN models (gameplay interaction, physiological and self-report data) were collected through survey experiments of children playing simple physical activity games designed on the Playware platform.

The adaptation mechanism employed here adjusts internal controls of the game (i.e. speed) during play to maximize the predicted entertainment value of the game derived from the interaction model. The mechanism's impact on physiology and the ANN physiological model is evaluated through a survey experiment. Results show that reported entertainment preferences correlate significantly with the entertainment values generated from the physiological model demonstrating the robustness of the model. Moreover, adaptation appears to generate two classes of physiological state which reveal different changes of the entertainment value - derived from the physiological affective model — and yield dissimilar preferences for the adaptation mechanism.

\section{Affective modeling through physiology in games}

The concept of affective gaming [9] has recently attracted significant attention within the affective computing community. Measurements of physiological quantities have been used extensively for emotion recognition in children and adults while playing games [4]. Correlations between physiological signals — galvanic skin response (GSR), jaw electromyography (EMG), respiration and cardiovascular measures - and reported adult user experiences in computer games have been examined by Mandryk et al. [14,15]. Working on the same basis, Ravaja et al. [19] examined whether the nature of the game opponent influences the physiological state of players. In addition, Hazlett's [7] work focused on the use of facial EMG to dis- 
tinguish positive and negative emotional valence during interaction with a racing video game. Moreover, Rani et al. [18] propose a methodology for detecting the anxiety level of the player and appropriately adjusting the level of challenge (e.g. speed) in the game of 'Pong'. On the same basis, a biocybernetic loop [4] that controls system adaptation through frustration (rather than anxiety) recognition is suggested by Gilleade et al. [11]. Physiological state (hearrate, galvanic skin response) prediction models have also been proposed for potential entertainment augmentation in computer games [16].

All of the studies referred to above use physiological measurements for capturing user experiences (e.g. "fun", engagement or excitement) applied within screen-based computer games. On the other hand, heart rate (HR) signals have been monitored to effect discrimination between children's exploration, problem-solving and play tasks [1]. Experiments with two-year old children further showed suppression of heart rate variability (HRV) during exploration, and solution of a puzzle, suggesting that the task demands for these two activities were greater than those during play [10]. Experiments by Yannakakis et al. [23, 26, 29] have distinguished those features of HR, skin conductance (SC) and blood volume pulse (BVP) signal recordings of children attributed to entertainment from those features that correspond to pure physical activity in action games played in interactive physical playgrounds. In those studies, highly accurate subjective models (predictors of entertainment preferences) of reported "fun" grounded in statistical features of physiological signal dynamics were constructed.

This study is innovative in that it explores the impact of an adaptive human-machine interactive system on physiology and affect. Physical interactive games are used as the initial test-bed evaluation scenario. To the best of the author's knowledge, there is no other study investigating closely related aspects of affective modeling and intelligent interaction. Given that very little is known about the efficacy of adaptation in terms of psychophysiological state [4], this paper provides some first insight into the interplay between adaptation and psychophysiology during physical interactive play. This work follows an implicit adaptation approach of the biocybernetic loop as presented in [4]: The game adapts according to a cognitive model of reported "fun" built on player-game interaction data (i.e. interaction model) and the impact of adaptivity on an accurate affective model built on physiology is investigated.

\section{Affective Model Construction}

The work described in this paper builds on quantitative user emotion preference models whose construction and evaluation has been fully described in the literature. For completeness, a brief recapitulation of the key points follows. The reader is referred to [26] for further details of the test-bed used and the entertainment (user) modeling methodology employed.

\subsection{Experimental Setup}

The test-bed game used for the experiments presented here is called 'Bug-Smasher'. The game is developed on a $6 \times 6$ square Playware [12] playground, comprising 36 tiles each incorporating processing power, communication, input (force pressure sensor) and output (light). The tile's dimensions are $21 \mathrm{~cm} \times 21 \mathrm{~cm} \times 4 \mathrm{~cm}$. During the game, different 'bugs' (colored lights) appear sequentially on the game surface and disappear again after a short period of time as a tile's light turns on and off respectively. The bug's position is picked randomly according to a predefined level of spatial diversity, measured by the entropy $(H)$ of the bugvisited tiles. The child's goal is to smash as many bugs as possible by stepping on the lighted tiles, thereby causing a force-sensor input to the tile. Screen-shots of the tile setup and the Bug-Smasher game are presented in Section 5.

In [26], physiological signals and children's preferences of entertainment were acquired for Bug-Smasher. Seventy two children aged from 8 to 10 years participated in the experiment reported. All participants were normal-weighted ${ }^{1}$, to minimize the effect of weight as a factor on physical interaction and playing experience. Heart Rate (HR), Blood Volume Pulse (BVP) and Skin Conductance (SC) were recorded in real-time and a number of statistical features of those signals were extracted. Children played two game variants for 90 seconds each; the two games differed in the levels of challenge (i.e. bug's speed) and curiosity (i.e. bugs' spatial diversity) factors identified by Malone [13]. For each completed pair of games, children reported their "fun" preference using a 2-alternative forced choice (2-AFC) protocol.

\subsection{Feature Selection and Preference Learning}

To construct a quantitative user preference model from the game pair data, the Sequential Forward Selection (SFS) feature selection technique is applied to select that subset of a set of candidate individual physiological signal features that allows the best-performing ANN model to be built. The SFS method is a bottom-up search procedure in which one feature is added at a time to the current feature set. The feature to be added is selected from the set of remaining candidate features such that the new feature set generates the maximum value of the performance function over all candidates for addition [2].

The key assumption in model construction is that the entertainment value $y$ of a given game — which models the subject's internal response to playing the game, that is, how much "fun" it is - is an unknown function of individ-

\footnotetext{
${ }^{1}$ Based on their body mass index lying between 18.5 and 25 .
} 


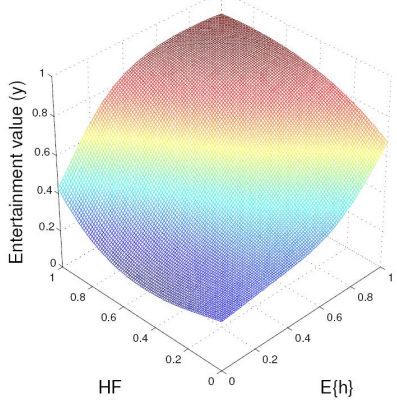

(a) $\sigma\{R R\}=0.0$

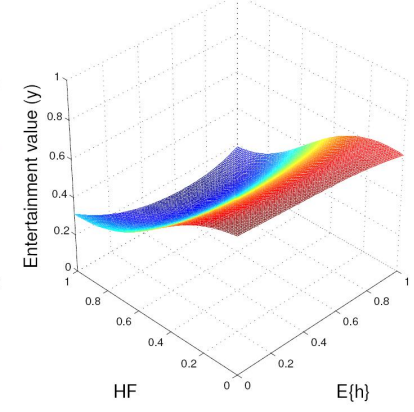

(b) $\sigma\{R R\}=1.0$
Figure 1. Evolved ANN, reported in [26], that yields the best classification accuracy on unknown data (89.29\%): ANN output $y$ (entertainment value) with regards to $\mathrm{HF}$ and $E\{h\}$ for two values of $\sigma\{R R\}(0.0,1.0)$.

ual physiological signal features which a machine learning mechanism can learn. The subject's expressed preferences constrain but do not specify the values of $y$ for individual games but it is assumed that the subject's expressed preferences are consistent.

The ANN representing the user preference model is constructed using a preference learning [3] approach in which a fully-connected ANN of fixed topology is evolved by a generational genetic algorithm (GA) [8] which uses a fitness function that measures the difference between the children's reported preferences of entertainment and the model output value $y$. The GA chromosome is a vector of ANN connection weights. The performance of an ANN model is measured through the average classification accuracy of the ANN in three independent runs using 3-fold crossvalidation on these training and validation data sets.

Experiments for finding the candidate feature subset yielding the highest ANN performance resulted [26] in a 3 -fold cross-validation performance of $79.76 \%$ (average of $75 \%, 75.00 \%$ and $89.29 \%$ ) when the ANN input (selected features from SFS) contains the average heart rate, $E\{h\}$, the standard deviation of inter-beat (RR) time intervals, $\sigma\{R R\}$, and the energy of the high frequency band (i.e. $(0.15,0.4] \mathrm{Hz})$ of heart rate variability, HF. The HF feature indicates high parasympathetic heart activity and is suppressed during mental or emotional stress $[5,22]$. The function between $\mathrm{HF}, E\{h\}, \sigma\{R R\}$ and the game's predicted entertainment value ( $y$ ) given by the highest performing ANN found is illustrated in Figure 1.

\section{Real-time Adaptation Mechanism}

The real-time adaptation mechanism presented here is introduced in [27] and the reader is referred to that study for all details covering the mechanism. Herein, the main elements of the mechanism are discussed; in particular, the game controllable features that are adjusted and the frequency of adaptation.

The idea behind real-time adaptation is to use a "fun" metric evaluation function (ANN user preference model) directly to enhance the entertainment provided by the game. The key to this is the observation that the ANN interaction user model [27] represents a (differentiable) functional relationship of game (controllable) features to an entertainment value $y$. It is therefore possible in principle to infer what changes to game features will cause an increase in the entertainment value of the game, and to adjust game parameters to make those changes. Thus a simple gradientascent mechanism is used to adjust the levels of challenge (i.e. speed) and curiosity (i.e. $H$ ) to maximize the reported entertainment value $y$ in real-time. Adjustments of speed and $H$ occur on the $45^{\text {th }}$, the $60^{\text {th }}$ and the $75^{\text {th }}$ second of the 90 second Bug-Smasher game.

\section{Adaptation experiment}

The mechanism described in the previous section was tested with Bug-Smasher. Two variants of the game were constructed: the static and the adaptive. The bugs' speed $(S)$ and spatial diversity $(H)$ for the static game were adjusted to the average of the values used in all Bug-Smasher experiments [24]. The adaptive game was initialized with the same values for speed and spatial diversity but the challenge and curiosity levels (states) were adjusted, according to measured player interaction, using the adaptation rules presented in [27] three times during the game: at 45, 60 and 75 seconds.

For the adaptation experiment, 24 naive normalweighted children (13 boys and 11 girls) aged 8 to 10 years are asked to play 4 games each on the Playware platform. The set of 4 games played comprised 2 games of static and 2 games of adaptive Bug-Smasher in all combinations. Thus, the number of children participating in the experiment is $4 \cdot C_{2}^{4}=24$, this being four times the required number of all combinations of 2 out of 4 games. Subjects play games in pairs and each time a pair of games is finished, the child is asked to choose among the following alternatives: (a) the first [second] game was more "fun" (see [20] for terminology used in experiments with children) than the second [first] game ( $c f$. 2-alternative forced choice); (b) both games were equally "fun" or (c) neither of the two games was "fun."

Note that children are not interviewed but are asked to fill in the comparison questionnaire above, minimizing interviewing [15] and experimenter expectancy effects [21]. Children complete a questionnaire after games 2, 3, and 4 , resulting in three "fun" comparisons (expressed preferences) between games $1-2,2-3$, and 3-4, for each child to report. That provides a total of 72 ( 24 children times 3 comparisons) "fun" comparisons. The 4-alternative forced 

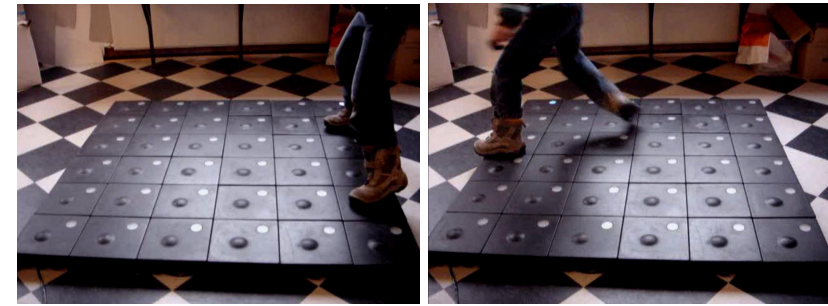

(a) Snapshot at the $5^{\text {th }}$ second of the (b) Snapshot at the $80^{\text {th }}$ second of game the game

Figure 2. Subject no. 6 playing the adaptive Bug-Smasher game that adjusts the level of curiosity $(H)$ and challenge $(S)$ in realtime. The adaptation rules during this game are: $\mathrm{H}-(45 \mathrm{sec}), \mathrm{H}$ $+(60 \mathrm{sec})$ and $\mathrm{H}+(75 \mathrm{sec})$. Note that subject no. 6 preferred the adaptive over the static game he played.

choice (4-AFC) protocol above is used since it offers several advantages for subjective entertainment capture: it minimizes the assumptions made about children's notions of "fun" and allows a fair comparison between the answers of different children while also making explicit the "no preference" cases.

As an example of a playing behavior, Figure 2 depicts screen-shots of the initial and final seconds of an adaptive game played by a subject that expressed a preference for the adaptive over the static game. This participant played the static game second and the specific adaptive game first. As seen in Fig 2(a) the game starts with low levels of curiosity corresponding to bugs that mainly appear at the right hand side of the topology. As the game proceeds the curiosity level is increased $(\mathrm{H}+)$ which results to bugs appearing in a less predictable manner in more tile positions of the topology (see Figure 2(b)).

\section{Results}

Given the experimental protocol there are 50 out of 72 "fun" comparisons between the static $(S)$ and the adaptive (A) Bug-Smasher. Within those 50 comparisons, 20 choices select the 'both games were equally fun' $(A=S)$ alternative and not a single choice the 'neither was fun' alternative. The first indicates the difficulty for the respective children of distinguishing between the two games whereas the latter shows that both games offered an enjoyable experience to all children participating in the experiment. In 18 and 12 out of the remaining 30 comparisons, children expressed a preference for the adaptive $(A \succ S)$ and the static ( $A \prec S$ ) game respectively. Even though not statistically significant, the percentage of children's preference for the adaptive game provide promise for the effectiveness of the adaptation mechanism as shown in [28].

\subsection{Affective Model and Reported Preferences}

Given the individual physiological signal features and the ANN affective model reported in [26], the $y$ values of the aforementioned 30 static and adaptive game pairs played are calculated via forward activation of the ANN model. Then the test statistic introduced in [25] is followed for validating the hypothesis that observed human judgement of entertainment correlates with the computed entertainment value, as far as the different game variants are concerned. The correlation value between the generated $y$ values and the reported entertainment preferences of the static-adaptive game pairs equals 0.7333 and its corresponding binomial distributed p-value is 0.002 . This suggests that the generated $y$ values correlate significantly with children's preferences and demonstrates the robustness of the affective model to efficiently predict entertainment preferences in the adaptation experimental study presented here. Even though this study includes physiological data sampled from adaptive games - on which the model is not trained - the model yields a statistically significant correlation with the expressed preferences of those games.

\subsection{Adaptivity and Physiology}

Herein the impact of the adaptation mechanism on children's physiology is investigated given the 30 staticadaptive pair values in which children expressed a clear preference for either the adaptive $(A \succ S)$ or the static ( $A \prec S$ ) game. This paper focuses on the three physiological signal features that constitute the input vector of the affective physiological ANN model: HF, $E\{h\}$ and $\sigma\{R R\}$. Subsequently the impact of adaptation on the entertainment value generated in those games is also investigated. The assumption made hereby is that each subject playing the same game variant (i.e. static game) twice will yield similar physiological indexes — inputs of the ANN model — and generate a similar entertainment value through the ANN model. This assumption is supported by a $t$-test for means of paired samples which demonstrates no significant difference (significance equals $5 \%$ in this paper) in $\mathrm{HF}(t=2.2033$, $P(T \leq t)=0.0634), E\{h\}(t=-0.8744, P(T \leq t)=$ $0.4108), \sigma\{R R\}(t=1.5852, P(T \leq t)=0.1569)$ and the entertainment value $(t=-1.5847, P(T \leq t)=0.1570)$ between the static games played by the same child.

As a first step, the differences between the three ANN input values generated during the adaptive game and the corresponding values generated at the static game played by each subject are calculated. These differences are illustrated in Figure 3(a) denoted as $\Delta \mathrm{HF}, \Delta E\{h\}$ and $\Delta \sigma\{R R\}$. A clear observation (see Figure $3(\mathrm{a})$ ) is the existence of two main clusters of static-adaptive game pairs. More data clusters can possibly be identified; however, $k$-means clustering [6] reveals that two $(k=2)$ classes are adequate to 


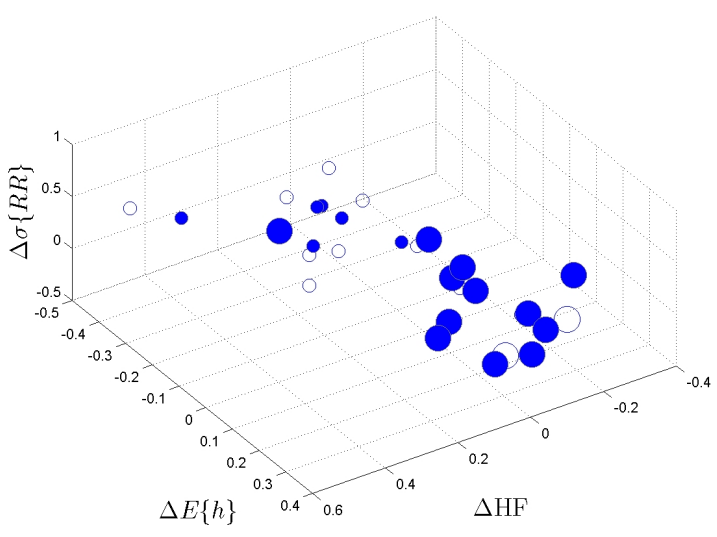

(a) Big and small circles represent increase and decrease of the $y$ value, respectively, due to adaptation.

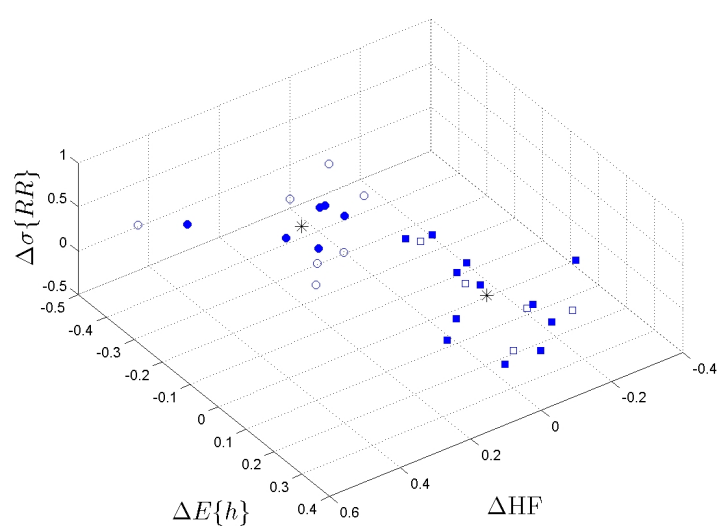

(b) Data clustered using K-means $(k=2)$. The two data clusters are represented by circles and squares while cluster centroids are represented by asterisks ('*').

Figure 3. ANN input value differences between the adaptive and the static game. Filled markers - circles (' $\bullet$ ') or squares (' $\boldsymbol{\square}$ ') - correspond to game pairs where the adaptive is preferred to the static game $(A \succ B)$. Empty markers - circles ('o') or squares (' $\square$ ') — correspond to game pairs where the static is preferred to the adaptive game $(A \prec B)$.

yield a clear distinction between the clusters of data. Note that the average quantization error in 1-means and 2-means clustering is 0.1519 and 0.0617 respectively.

Within the first class of game pairs (right cluster centroid of Figure 3(b)) we meet children whose HF energy and standard deviation of the RR intervals are either slightly increased or decreased due to adaptation: $\Delta \mathrm{HF}<0.1$ and $\sigma\{R R\}<0.1$. Furthermore their average heart rate is increased $E\{h\}>0.0$ when the adaptive game is played. This corresponds to a constantly (decreased $\sigma\{R R\}$ ) more energetic (increased $E\{h\}$ ) play of higher emotional or mental load (decreased HF) which is reported as entertaining for the majority (12 out of 17) of children of this class.

The second class (left cluster centroid of Figure 3(b)) corresponds to children with increased HF energy $(\Delta \mathrm{HF}>$ $0.0)$ and standard deviation of RR intervals $(\Delta \sigma\{R R\}>$ $0.1)$ and decreased average heart rate $(\Delta E\{h\}<0.0)$ due to adaptation. The adaptive game appears to generate a more dynamic (increased $\sigma\{R R\}$ ) play that involves lower mental or emotional load (increased HF) and lower physical effort (decreased $E\{h\}$ ). Children of this class have a slight preference for the static over the adaptive game instead since the static game is chosen in 6 out of 13 game pair instances. This generates the assumption that a game which forces children towards a more dynamic play and generates lower mental or emotional load combined with lower physical effort appears to be preferred by children. The validity of this assumption is supported by the observation that $y$ is increased due to adaptation in only 1 out of 13 instances of the second cluster. On the other hand, $y$ is increased due to adaptation in 13 out of 17 game pair instances of the first cluster (see Figure 3(a)).

\section{Conclusions}

This paper investigated the impact of game adaptation on entertainment preferences of children through a constructed affective model for predicting user entertainment preferences. An evaluation survey experiment using Playware physical interactive game variants is designed and shows that the affective model built on static game data collected from earlier studies correlates significantly with entertainment preferences expressed in this evaluation study. Results demonstrate the robustness and generality of the model in predicting reported entertainment preferences even in adaptive physical interactive games.

The relationship between adaptation and generated physiological state reveals two classes of subjects. The first class corresponds to a constantly more energetic play of higher emotional or mental load. The second class corresponds to a more dynamic play involving lower mental or emotional load and lower physical effort. The majority of children within the first class prefer the adaptive game and the ANN model generates a higher entertainment value, $y$, for the majority of the adaptive games of that class. On the other hand, children of the second class do not express an obvious preference for the adaptive game while the corresponding generated $y$ values are lower in the vast majority of game pair instances.

A fuller analysis of the adaptation mechanism will be required to identify causes leading to the adaptive games of the second class. An initial study has already shown that maladjustments of internal game controls (challenge, curiosity) have an impact on the performance of the adaptation mechanism and furthermore on expressed preferences [28]. 
On that basis, adaptation maladjustments might also lead to physiological states, such as the state of children of the second class, that affect children's preferences of adaptation. Moreover the impact of each in-game control adjustment (occurring on the $45^{\text {th }}$, the $60^{\text {th }}$ and the $75^{\text {th }}$ second of the game) on physiology would reveal more details on the relation between adaptation and its effect to physiology in physical play. Towards this aim more physiological signal features could be explored and the impact of adaptation on those features would provide further insight into the adaptation-physiology relationship.

\section{References}

[1] C. Hutt. Exploration and play, in Play and learning, pages 175-194. Gardner Press: New York, 1979.

[2] P. Devijver and J. Kittler. Pattern Recognition - A Statistical Approach. Prentice-Hall, Engelwood cliffs, NJ, 1982.

[3] J. Doyle. Prospects for preferences. Computational Intelligence, 20(2):111-136, May 2004.

[4] S. H. Fairclough. Fundamentals of physiological computing. Interact. Comput., 21(1-2):133-145, 2009.

[5] J. J. Goldberger, S. Challapalli, R. Tung, M. A. Parker, and A. H. Kadish. Relationship of heart rate variability to parasympathetic effect. Circulation, 103:1977-1983, 2001.

[6] R. M. Gray. Vector quantization. IEEE ASSP Magazine, pages 4-29, 1984.

[7] R. L. Hazlett. Measuring emotional valence during interactive experiences: boys at video game play. In $\mathrm{CHI}$ '06: Proceedings of the SIGCHI conference on Human Factors in computing systems, pages 1023-1026, New York, NY, USA, 2006. ACM Press.

[8] J. H. Holland. Adaptation in Natural and Artificial Systems. University of Michigan Press, Ann Arbor, MI, 1975.

[9] E. Hudlicka. Affective game engines: Motivation and requirements. In Proceedings of the 4th Int. Conf. on the Foundations of Digital Games, 209.

[10] M. Hughes and C. Hutt. Heart-rate correlates of childhood activities: play, exploration, problem-solving and daydreaming. American Psychologist, 8(4):253-263, 1979.

[11] A. D. K. Gilleade and J. Allanson. Affective Videogames and Modes of Affective Gaming: Assist Me, Challenge Me, Emote Me. In Proceedings of DIGRA'05, 2005.

[12] H. H. Lund, T. Klitbo, and C. Jessen. Playware technology for physically activating play. Artifical Life and Robotics Journal, 9(4):165-174, 2005.

[13] T. W. Malone. What makes computer games fun? Byte, 6:258-277, 1981.

[14] R. L. Mandryk and M. S. Atkins. A Fuzzy Physiological Approach for Continuously Modeling Emotion During Interaction with Play Environments. International Journal of Human-Computer Studies, 65:329-347, 2007.

[15] R. L. Mandryk, K. M. Inkpen, and T. W. Calvert. Using Psychophysiological Techniques to Measure User Experience with Entertainment Technologies. Behaviour and Information Technology (Special Issue on User Experience), 25(2):141-158, 2006.
[16] S. McQuiggan, S. Lee, and J. Lester. Predicting User Physiological Response for Interactive Environments: An Inductive Approach. In Proceedings of the $2^{\text {nd }}$ Artificial Intelligence for Interactive Digital Entertainment Conference, pages 6065, 2006.

[17] A. T. Pope, E. H. Bogart, and D. S. Bartolome. Biocybernetic system evaluates indices of operator engagement in automated task. Biological Psychology, 40:187-195, 1995.

[18] P. Rani, N. Sarkar, and C. Liu. Maintaining optimal challenge in computer games through real-time physiological feedback. In Proceedings of the $11^{\text {th }}$ International Conference on Human Computer Interaction, 2005.

[19] N. Ravaja, T. Saari, M. Turpeinen, J. Laarni, M. Salminen, and M. Kivikangas. Spatial Presence and Emotions during Video Game Playing: Does It Matter with Whom You Play? Presence Teleoperators \& Virtual Environments, 15(4):381392, 2006.

[20] J. Read, S. MacFarlane, and C. Cassey. Endurability, engagement and expectations. In Proceedings of International Conference for Interaction Design and Children, 2002.

[21] R. Rosenthal. Covert communication in laboratories, classrooms, and the truly real world. Current Directions in Psychological Science, 12(5):151-154, 2003.

[22] D. W. Rowe, J. Sibert, and D. Irwin. Heart Rate Variability: Indicator of User State as an aid to Human-Computer Interaction. In Proceedings of Conference on Human Factors in Computing Systems, pages 480-487, 1998.

[23] G. N. Yannakakis and J. Hallam. Entertainment Modeling in Physical Play through Physiology beyond Heart-Rate. In A. Paiva, R. Prada, and R. Picard, editors, Proceedings of the International Conference on Affective Computing and Intelligent Interaction, LNCS 4738, pages 256-267, Lisbon, Portugal, September 2007. Springer-Verlag.

[24] G. N. Yannakakis and J. Hallam. Game and Player Feature Selection for Entertainment Capture. In Proceedings of the IEEE Symposium on Computational Intelligence and Games, pages 244-251, Hawaii, USA, April 2007. IEEE.

[25] G. N. Yannakakis and J. Hallam. Towards Optimizing Entertainment in Computer Games. Applied Artificial Intelligence, 21:933-971, 2007.

[26] G. N. Yannakakis and J. Hallam. Entertainment Modeling through Physiology in Physical Play. International Journal of Human-Computer Studies, 66:741-755, October 2008.

[27] G. N. Yannakakis and J. Hallam. Real-time Adaptation of Augmented-Reality Games for Optimizing Player Satisfaction. In Proceedings of the IEEE Symposium on Computational Intelligence and Games, pages 103-110, Perth, Australia, December 2008. IEEE.

[28] G. N. Yannakakis and J. Hallam. Real-time Game Adaptation for Optimizing Player Satisfaction. IEEE Transactions on Computational Intelligence and AI in Games, 1(2):121133, June 2009.

[29] G. N. Yannakakis, J. Hallam, and H. H. Lund. Entertainment Capture through Heart Rate Activity in Physical Interactive Playgrounds. User Modeling and User-Adapted Interaction, Special Issue: Affective Modeling and Adaptation, 18(1-2):207-243, February 2008. 